\title{
Gastrointestinal relapse of multiple myeloma and sustained response to lenalidomide: a case report
}

\author{
Patrick R Benusiglio ${ }^{*}$, Thomas A McKee², Xavier Montet ${ }^{3}$, Jean-Marc Dumonceau ${ }^{4}$, Laurence Favet ${ }^{1}$, \\ Anne-Claude George ${ }^{1}$ and Pierre-Yves Dietrich ${ }^{1}$
}

\begin{abstract}
Introduction: Gastrointestinal relapse in patients with multiple myeloma is very rare and, when reported, always associated with a poor prognosis.

Case presentation: We describe the case of a 71-year-old Caucasian man who presented with life-threatening hematemesis and melena due to a digestive relapse of his multiple myeloma. Despite the active hemorrhage, we initiated a third-line treatment with lenalidomide. The response was spectacular and long-lasting.

Conclusions: Clinicians must consider digestive tract involvement in myeloma patients presenting with a gastrointestinal hemorrhage. Furthermore, myeloma patients do benefit from novel oral drugs, even when they are critically ill.
\end{abstract}

\section{Introduction}

The involvement of the gastrointestinal tract years after an initial diagnosis of multiple myeloma (MM) is exceptional and, when reported, always associated with a poor prognosis [1-4]. We report the case of a 71-year-old man with MM who had been heavily pre-treated and who presented with hematemesis and melena due to a gastrointestinal relapse of his disease. The bleeding lasted for over two weeks and soon became life-threatening. Despite the active hemorrage, we initiated a third-line treatment with lenalidomide. The response was spectacular.

\section{Case presentation}

We report the case of a 71-year-old Caucasian diabetic man with severe diabetic neuropathy who was diagnosed with stage IIIA IgG $\lambda$ MM in 2004. He was initially treated with three cycles of vincristine, doxorubicin and dexamethasone, followed by high-dose melphalan and autologous stem-cell transplantation, which resulted in a partial response. His monoclonal IgG had dropped from $65 \mathrm{~g} / \mathrm{L}$ before treatment to $11 \mathrm{~g} / \mathrm{L}$ after transplantation. In 2007, a second-line chemotherapy treatment

\footnotetext{
* Correspondence: Patrick.Benusiglio@hcuge.ch

'Centre for Oncology, Geneva University Hospital, 4 rue Gabrielle Perret-

Gentil, 1211 Geneva 14, Switzerland

Full list of author information is available at the end of the article
}

(melphalan and prednisone, six cycles) for a relapse characterized by diffuse spinal involvement and an increase in monoclonal IgG $(22 \mathrm{~g} / \mathrm{L})$ stabilized the disease. His neuropathy had precluded treatment with thalidomide or bortezomib. Eight months after the second-line chemotherapy, an irradiation to the T10-L1 vertebrae (30 Gy) was undertaken for symptomatic, localized bone involvement. Ten months later, an increase in his level of IgG $(34 \mathrm{~g} / \mathrm{L})$, combined with widespread bone pain and a worsening of his general condition, led to the introduction of high-dose steroids.

After a week of steroid treatment, he was admitted to our hospital for the first time with chest pain and dyspnea. He was febrile $\left(38.4^{\circ} \mathrm{C}\right)$ and his inflammatory parameters were increased (C-reactive protein $91 \mathrm{mg} / \mathrm{L}$ ). A urinary test for the Legionella pneumophila antigen was positive and a computed tomography $(\mathrm{CT})$ scan showed trilobar consolidation and a bilateral pleural effusion. A heterogeneous solid mass extending from the retroperitoneal to the peritoneal spaces (Figure 1A, B) provided evidence for the progression of the MM. At the time, priority was given to the treatment of the pulmonary infection and he recovered after three weeks of oral levofloxacin.

Shortly after the antibiotic therapy was discontinued, he presented with sudden hematemesis and melena, requiring fifteen $500 \mathrm{ml}$ units of packed red cells, in

\section{Ciomed Central}



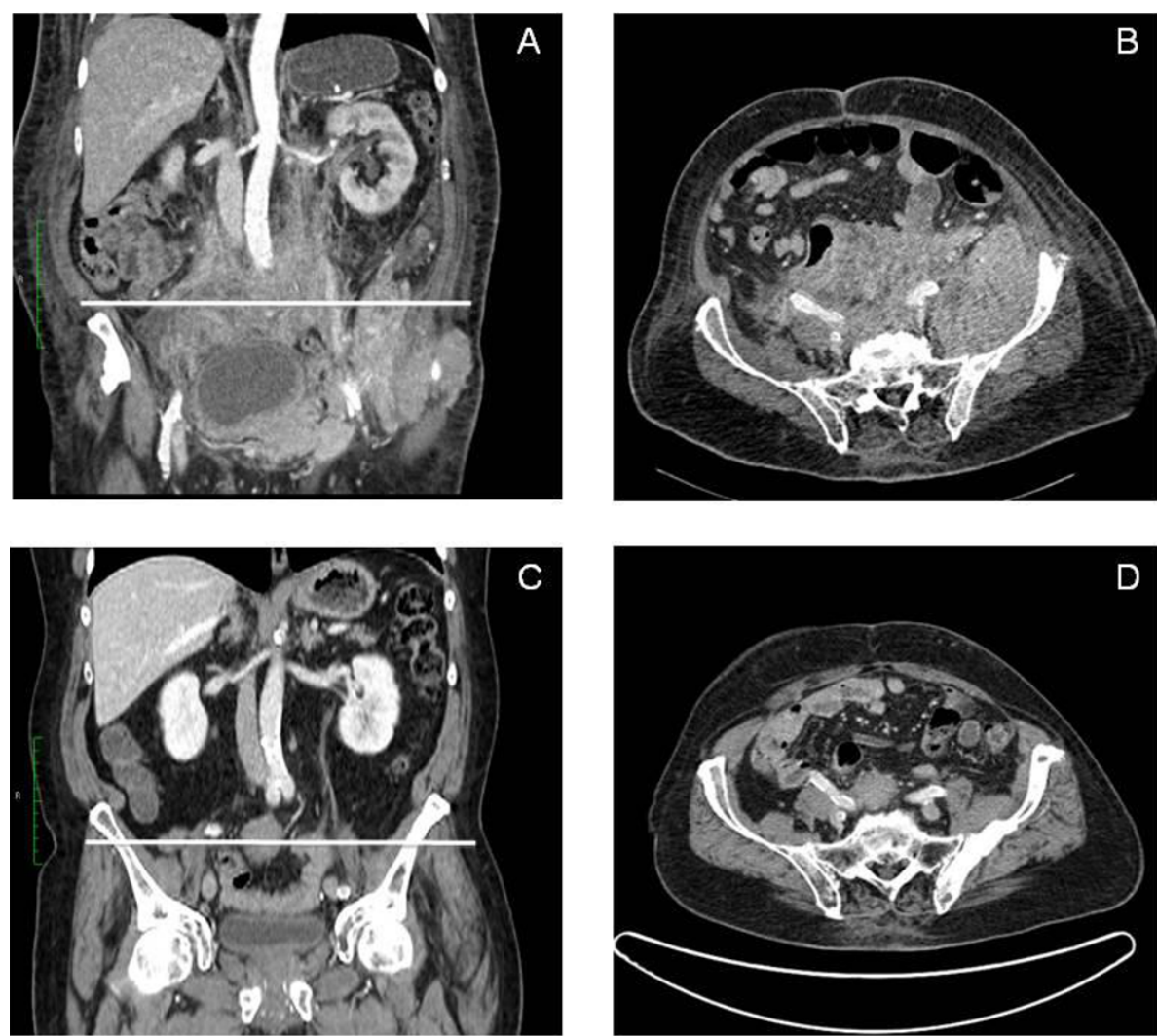

Figure $1 \mathrm{CT}$ of the abdomen before $(\mathrm{A}, \mathrm{B})$ and after $(\mathrm{C}, \mathrm{D})$ four months of treatment with lenalidomide

total, over a period of twenty days. His platelet count and coagulation parameters were normal. A bleeding, ulcerated jejunal mass was revealed by an upper gastrointestinal endoscopy (Figure 2) and biopsies showed an infiltration of the intestinal mucosa by neoplastic plasma

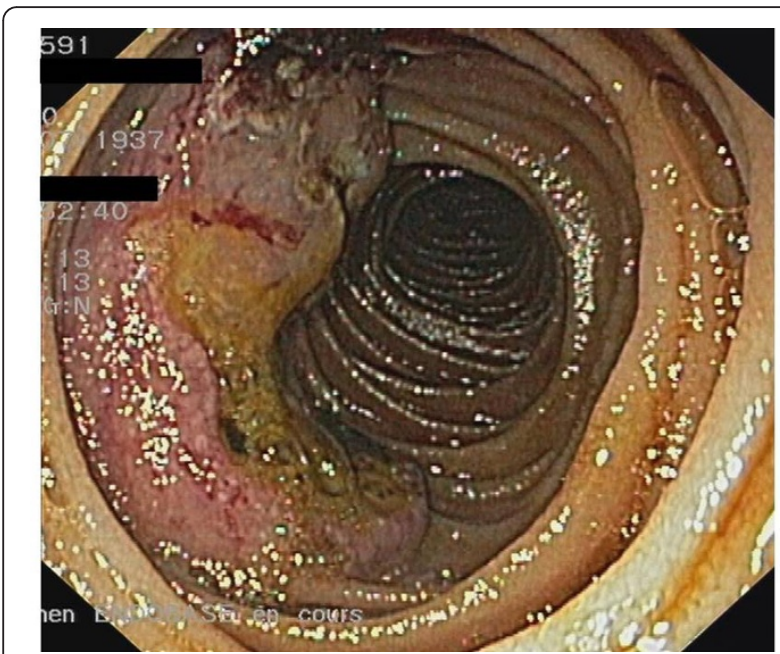

Figure 2 Jejunal mass as seen on the upper gastrointestinal endoscopy cells producing monoclonal $\lambda$ light chains (Figure 3A,B). Despite the active bleeding, a third-line therapy with lenalidomide ( $25 \mathrm{mg}$ daily) and dexamethasone (40 $\mathrm{mg}$ once-weekly) was initiated; the lenalidomide was given in three-week cycles followed by a one-week break $[5,6]$. An excellent response was achieved after the first cycle: his paraprotein levels dropped to $10 \mathrm{~g} / \mathrm{L}$ and there was no recurrence of the hematemesis or melena. His general condition improved rapidly and he was discharged after the second cycle had commenced. A repeat CT four months later showed a dramatic shrinkage of the retroperitoneal mass (Figure 1C,D). This response lasted for a total of 10 months and resulted in an excellent

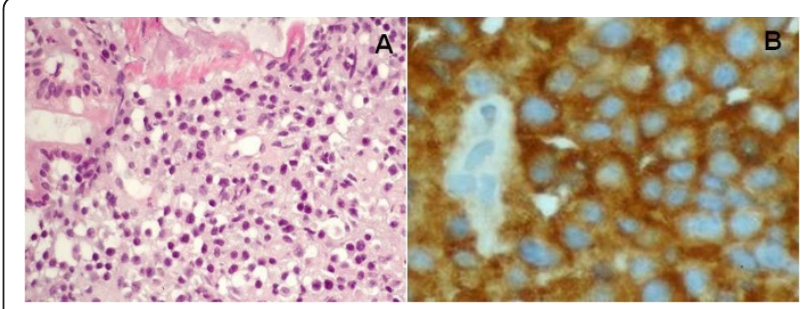

Figure 3 Jejunal mucosa infiltrated by multiple myeloma. A magnification $\times 200$, hematoxylin and eosin. B: magnification $\times 400$, $\lambda$ chains. 
quality of life for the patient during the whole period. He declined further treatment at retroperitoneal progression and died a few weeks later.

\section{Discussion}

Gastrointestinal involvement in MM is very rare. It most often occurs in the context of an isolated, primary, extramedullary plasmacytoma [7]. Patients with newlydiagnosed MM rarely present with symptoms related to gastrointestinal involvement [8]. A gastrointestinal relapse in patients with long-term MM, such as that observed in the case of our patient, is exceptional. Amongst a total of 553 patients with MM included in two large European studies, 87 experienced an extramedullary relapse but none of these involved the gastrointestinal tract $[1,2]$. Only one out of six extramedullary relapses reported by a North American Institution involved the gastrointestinal tract [3]. All of these cases had a poor prognosis, with a maximal survival rate of 106 days from diagnosis. Finally, Dawson et al. reported the case of a 60-year-old patient with MM with hematemesis, melena and gastroduodenal mucosal lesions [4]. The patient died two weeks after presentation. The gastrointestinal lesions were not biopsied, but their myelomatous nature was likely, as a biopsy of a right breast mass provided pathological evidence of an extramedullary relapse.

\section{Conclusion}

Our case report is well documented and highly informative. It reminds us that, in addition to much more common causes (for example, ulcers), clinicians must consider digestive tract involvement in patients with MM presenting with a gastrointestinal hemorrhage. It also shows that patients with MM who have been heavily pre-treated can benefit from novel drugs, even when they are critically ill. We suggest that the major clinical improvement has to be linked to lenalidomide, since high-dose steroids had been ineffective in this case. Our patient's recovery and the drop in his monoclonal IgG were very rapid. This effect of lenalidomide has already been observed in other life-threatening situations associated with MM, such as severe renal impairment or high-output heart failure secondary to intramedullary arteriovenous fistulas $[9,10]$. Finally, it should be emphasized that a response to this oral drug was obtained despite active bleeding in the upper digestive tract.

\section{Consent}

Written informed consent was obtained from the patient's next-of-kin for publication of this case report and any accompanying images. A copy of the written consent is available for review by the Editor-in-Chief of this journal.

\section{Author details}

'Centre for Oncology, Geneva University Hospital, 4 rue Gabrielle PerretGentil, 1211 Geneva 14, Switzerland. 'Department of Pathology, Geneva University Hospital, 4 rue Gabrielle Perret-Gentil, 1211 Geneva 14 Switzerland. ${ }^{3}$ Department of Radiology, Geneva University Hospital, 4 rue Gabrielle Perret-Gentil, 1211 Geneva 14, Switzerland. ${ }^{4}$ Department of Gastroenterology, Geneva University Hospital, 4 rue Gabrielle Perret-Gentil, 1211 Geneva 14, Switzerland.

\section{Authors' contributions}

PRB, JMD, LF, ACG and PYD were directly involved in the management of the patient. PRB wrote the manuscript with support from TAM and PYD. TAM and XM reviewed and interpreted the pathology slides and CT scan images, respectively. All authors read and approved the final manuscript.

\section{Competing interests}

The authors declare that they have no competing interests.

Received: 5 August 2010 Accepted: 19 March 2011

Published: 19 March 2011

\section{References}

1. Damaj G, Mohty M, Vey N, Dincan E, Bouabdallah R, Faucher C, Stoppa AM, Gastaut JA: Features of extramedullary and extraosseous multiple myeloma: a report of 19 patients from a single center. Eur I Haematol 2004, 73:402-406.

2. Zeiser R, Deschler B, Bertz H, Engelhardt M: Extramedullary vs medullary relapse after autologous or allogeneic hematopoietic stem cell transplantation (HSCT) in multiple myeloma (MM) and its correlation to clinical outcome. Bone Marrow Transplant 2004, 34:1057-1065.

3. Cerny J, Fadare O, Hutchinson L, Wang SA: Clinicopathological features of extramedullary recurrence/relapse of multiple myeloma. Eur I Haematol 2008, 81:65-69.

4. Dawson MA, Polizzotto MN, Gordon A, Roberts SK, Spencer A: Extramedullary relapse of multiple myeloma presenting as hematemesis and melena. Nat Clin Pract Oncol 2006, 3:223-226.

5. Weber DM, Chen C, Niesvizky R, Wang M, Belch A, Stadtmauer EA, Siegel D, Borrello I, Rajkumar SV, Chanan-Khan AA, Lonial S, Yu Z, Patin J, Olesnyckyj M, Zeldis JB, Knight RD, Multiple Myeloma (009) Study Investigators: Lenalidomide plus dexamethasone for relapsed multiple myeloma in North America. N Engl J Med 2007, 357:2133-2142.

6. Rajkumar SV, Jacobus S, Callander NS, Fonseca R, Vesole DH, Williams ME, Abonour R, Siegel DS, Katz M, Greipp PR, Eastern Cooperative Oncology Group: Lenalidomide plus high-dose dexamethasone versus lenalidomide plus low-dose dexamethasone as initial therapy for newly diagnosed multiple myeloma: an open-label randomised controlled trial. Lancet Oncol 2010, 11(1):29-37.

7. Alexiou C, Kau RJ, Dietzfelbinger H, Kremer M, Spiess JC, Schratzenstaller B, Arnold W: Extramedullary plasmacytoma: tumor occurrence and therapeutic concepts. Cancer 1999, 85:2305-2314.

8. Herbst A, Renner SW, Ringenberg QS, Fass R, Krouse RS: Multiple myeloma presenting with a colonic obstruction and bony lesions: a clinical dilemma. J Clin Oncol 2008, 26:5645-5647

9. Ludwig H, Zojer N: Renal recovery with lenalidomide in a patient with bortezomib-resistant multiple myeloma. Nat Rev Clin Oncol 2010, 7(5):289-294.

10. Robin J, Fintel B, Pikovskaya O, Davidson C, Cilley J, Flaherty J: Multiple myeloma presenting with high-output heart failure and improving with anti-angiogenesis therapy: two case reports and a review of the literature. J Med Case Reports 2008, 2:229.

doi:10.1186/1752-1947-5-110

Cite this article as: Benusiglio et al:: Gastrointestinal relapse of multiple myeloma and sustained response to lenalidomide: a case report. Journal of Medical Case Reports 2011 5:110. 